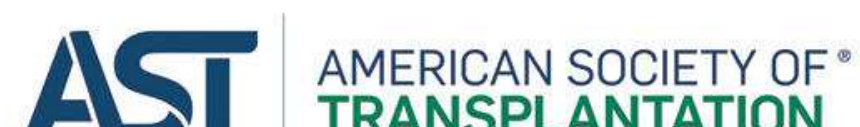 TRANSPLANTATION
}

\section{COMPREHENSIVE}

\section{TRAINEE}

\section{CURRICULUM}

\section{COMPREHENSIVE TRAINEE CURRICULUM}

\section{WATCH 52 ONLINE LESSONS INCLUDING:}

- Adult and Pediatric Liver

- Adult Cardiac and Pulmonary

- Abdominal Transplant Surgery
- Cardiothoracic Transplant Surgery

- Adult and Pediatric Kidney

- Transplant Pharmacy
Solid organ transplantation is a multidisciplinary field, leading to a diverse community of professionals within the AST. As a result, it is often necessary for trainees to have extensive knowledge of all areas of transplantation-not just their specialty.
Check out this brand new resource, meant to supplement the training trainees and fellows receive at their university or hospital.

\$50 members / \$200 non-members 


\title{
Dominance of free wall radial motion in global right ventricular function of heart transplant recipients
}

\author{
Bálint Károly Lakatos ${ }^{1}$ (D) | ${\text { Márton } \text { Tokodi }^{1} \text { | Alexandra Assabiny }}^{1}$ | \\ Zoltán Tősér $^{2}$ | Annamária Kosztin ${ }^{1}$ | Alexandra Doronina ${ }^{1}$ | Kristóf Rácz ${ }^{3}$ | \\ Kinga Bianka Koritsánszky ${ }^{3}$ | Viktor Berzsenyi ${ }^{3}$ | Endre Németh ${ }^{3}$ (D) | \\ Balázs Sax ${ }^{1}$ | Attila Kovács ${ }^{1}$ (D) | Béla Merkely ${ }^{1}$ (D)
}

${ }^{1}$ Heart and Vascular Center, Semmelweis University, Budapest, Hungary

${ }^{2}$ Argus Cognitive, Inc., Dover, DE, USA

${ }^{3}$ Department of Anesthesiology and Intensive Therapy, Semmelweis University, Budapest, Hungary

Correspondence

Attila Kovács, MD, PhD, Heart and Vascular Center, Semmelweis University, Budapest, Hungary.

Email: attila.kovacs@cirg.hu

Funding information

This work was supported by the National Research, Development and Innovation Office NKFIH of Hungary ("National Heart Program" NVKP_16-1-2016-0017; BM) and by the Arrhythmia Research Foundation.

\begin{abstract}
Assessment of right ventricular (RV) function using conventional echocardiography might be inadequate as the radial motion of the RV free wall is often neglected. Our aim was to quantify the longitudinal and the radial components of RV function using three-dimensional (3D) echocardiography in heart transplant (HTX) recipients. Fiftyone HTX patients in stable cardiovascular condition without history of relevant rejection episode or chronic allograft vasculopathy and 30 healthy volunteers were enrolled. RV end-diastolic (EDV) volume and total ejection fraction (TEF) were measured by 3D echocardiography. Furthermore, we quantified longitudinal (LEF) and radial ejection fraction (REF) by decomposing the motion of the RV using the ReVISION method. RV EDV did not differ between groups (HTX vs control; $96 \pm 27$ vs $97 \pm 2 \mathrm{~mL}$ ). In HTX patients, TEF was lower, however, tricuspid annular plane systolic excursion (TAPSE) decreased to a greater extent (TEF: $47 \pm 7$ vs $54 \pm 4 \%$ [-13\%], TAPSE: $11 \pm 5$ vs $21 \pm 4 \mathrm{~mm}$ [-48\%], $P<.0001)$. In HTX patients, REF/TEF ratio was significantly higher compared to LEF/TEF (REF/TEF vs LEF/TEF: $0.58 \pm 0.10$ vs $0.27 \pm 0.08$, $P<.0001)$, while in controls the REF/TEF and LEF/TEF ratio was similar $(0.45 \pm 0.07$ vs $0.47 \pm 0.07$ ). Current results confirm the superiority of radial motion in determining RV function in HTX patients. Parameters incorporating the radial motion are recommended to assess RV function in HTX recipients.
\end{abstract}

KEYWORDS

echocardiography, heart transplantation, right ventricle

\section{1 | INTRODUCTION}

Current heart failure guidelines strongly emphasize the importance and effectiveness of heart transplantation (HTX) in the management of end-stage heart failure. ${ }^{1,2}$ HTX provides excellent long-term survival and improved functional status in the majority of these patients with an 85\% 1-year and 73\% 5-year survival rate. ${ }^{3}$ However, early postoperative mortality is still significant, having right ventricular (RV) failure as one of the major determinants. ${ }^{4,5}$ This fact necessitates an accurate evaluation of RV function, while still little is known about the adaptive and/or maladaptive RV functional changes attributable to HTX both in the early and in the late postoperative period.

Echocardiography is the first line non-invasive method to measure RV function in HTX recipients; however, conventional techniques do 
have limitations. Parameters used in everyday practice refer predominantly to the longitudinal shortening of the chamber and generally suggest reduced function even in the absence of other clinical signs of right heart failure. Recent studies using cardiac MRI or threedimensional (3D) echocardiography reported normal RV ejection fraction along with poor correlation with routine parameters of RV longitudinal shortening in HTX patients. ${ }^{6,7}$ These findings indicate the presence of a functional shift, where conventional measurements are not able to accurately evaluate RV function. Therefore, current European Association of Cardiovascular Imaging guideline also recommends to report RV functional parameters that do not exclusively measure longitudinal shortening in HTX patients. ${ }^{4}$ To date, no established method was available to characterize the altered motion pattern of the RV and to quantify the importance of the radial motion of the free wall ("bellows" effect).

Accordingly, our aim was to determine the relative contribution of longitudinal and radial wall motions to global RV function in $\mathrm{HTX}$ recipients to confirm the dominance of radial motion using 3D echocardiography.

\section{PATIENTS AND METHODS}

\section{1 | Patient population}

In the time frame of December 2014 to January 2017, we have retrospectively collected those echocardiograms of HTX recipients followed-up by our Center, where transthoracic 3D datasets were acquired suitable for further analysis $(n=66)$. Those patients were included, who were already discharged from intensive care unit after HTX or arrived to regular follow-up visit. Exclusion criteria were (i) hemodynamic instability and/or need for inotropic agents; (ii) previous rejection $\geq I S H L T$ grade $2 R$ or $\geq$ pAMR2; (iii) postoperative need for ventricular assist device; (iv) severe tricuspid insufficiency or any severe valvular disease; (v) non-sinus rhythm on ECG; (vi) diagnosis of chronic allograft vasculopathy; (vii) suboptimal 3D echocardiographic image quality (inadequate visualization of the entire RV endocardial surface inclusive of RV outflow tract-confirmed also on short-axis planes-and/or the presence of stitching artifacts). Finally, 51 patients have been entered into current analysis. An age- and gender-matched control population ( $n=30$ ) was selected with a normal echocardiographic report and without any known cardiovascular or other diseases and free from any medication using our existing database of healthy volunteers.

\section{2 | Basic demographic, medical history, and perioperative data collection}

To create a relevant database, medical history, preoperative, intraoperative, and follow-up data of each patient were collected using the in-hospital electronic medical records. Anthropometric, blood pressure, and heart rate values were determined at time-point of the analyzed echocardiogram in both groups.

\section{3 | Echocardiography}

Our standard protocol consisted of two-dimensional (2D), M-mode, color Doppler, and spectral Doppler acquisitions from parasternal, apical, and subxyphoid approaches based on current recommendations. ${ }^{8,9}$ Grade of tricuspid regurgitation was assessed according to current guideline. ${ }^{10}$ Three-dimensional datasets were acquired from apical approach. ECG-gated full-volume datasets reconstructed from four or six cardiac cycles optimized for the left ventricle or the RV (Philips EPIQ 7G system, X5-1 transducer; Philips Healthcare, Best, The Netherlands) were further analyzed. Left ventricular 3D datasets were processed using a commercially available semi-automated software (4D LV-Analysis 3; TomTec Imaging GmbH, Unterschleissheim, Germany). Beyond end-diastolic-, end-systolic-, stroke volume, and ejection fraction, 3D global longitudinal strain was also calculated. By contouring the left ventricular epicardial surface on multiple short- and long-axis planes, left ventricular mass was also measured. Volumetric and mass parameters were indexed to body surface area (BSA) calculated by the Mosteller formula. ${ }^{11}$

\subsection{D RV analysis}

Off-line analysis was performed by a single experienced operator using a commercially available, semi-automated software (4D RVFunction 2, TomTec Imaging $\mathrm{GmbH}$ ) to quantify RV end-diastolic (EDV), end-systolic (ESV) volumes, stroke volume (SV), and total ejection fraction (TEF). Automatically generated RV endocardial contour was manually corrected on multiple short- and long-axis planes throughout the entire cardiac cycle. Furthermore, this software automatically measures RV basal short-axis diameter, RV mid short-axis diameter, RV length, tricuspid annular plane systolic excursion (TAPSE), fractional area change (FAC), 2D free wall longitudinal strain (LS), and septal longitudinal strain on the standard apical four-chamber view derived from the 3D dataset. The created 3D model was exported volume-by-volume throughout the cardiac cycle and analyzed further by our custom-made method (Right Ventrlcular Separate wall motlon quantificatiON-ReVISION method-freely available for scientific purposes at www.revisionmethod.com). In brief, the wall movements of the exported RV model are decomposed in a vertex-based manner. The volumes of the models accounting for only one direction were calculated at each time frame using the signed tetrahedron method. By the decomposition of the 3D model's motion along the three orthogonal, anatomically relevant axes, volume loss attributable to either longitudinal, radial or anteroposterior wall motions could be separately quantified. ${ }^{12,13}$ Thus, longitudinal (LEF), radial (REF), and anteroposterior (AEF) ejection fraction and their ratio to TEF (LEF/ TEF, REF/TEF, AEF/TEF, respectively) could be expressed as a measure of the relative contribution of the given wall motion direction.

\section{5 | Statistical analysis}

Data are presented as mean $\pm \mathrm{SD}$ or median (interquartile range) Shapiro-Wilk test was used to test normal distribution. Based on that, 
unpaired Student's t-test or Mann-Whitney U-test was used to compare groups. To compare categorical variables, chi-square test was applied. To compare the three wall motion component's relative contribution, one-way ANOVA was used followed by Tukey's post hoc test. Pearson's or Spearman's test was performed for correlation analysis as appropriate. The intraobserver and interobserver reproducibility were evaluated using Lin's concordance correlation coefficient. A non-echocardiographer investigator randomly selected 10 HTX patients and further five healthy controls and exported these studies anonymized. The main operator reconstructed the 3D RV models again to assess intraobserver variability compared to the original measurements, while a second experienced operator also performed the measurements to assess interobserver variability. Then, the fully automated ReVISION method was applied on the three subset of 3D models, and reproducibility of ESV values with either only longitudinal or only radial motion component enabled was calculated. $P$ values $<.05$ were considered significant.

\section{RESULTS}

Demographic characteristics of the study groups are shown in Table 1. The mean age of the predominantly male HTX patients was 52 years. The age- and gender-matched control group did not show any statistically significant difference in terms of height, weight, body mass index, BSA, systolic, and diastolic blood pressure compared to the HTX group (Table 1). HTX patients had significantly higher heart rate attributable to the denervation of the heart. The bicaval surgical technique was used in every patient.

Left ventricular end-diastolic-, end-systolic volumes, and stroke volume along with their BSA-indexed values showed no difference between the study groups (Table 1). Left ventricular ejection fraction and global longitudinal strain were also similar, excluding the presence of left ventricular systolic dysfunction. There was a trend toward significance in terms of higher left ventricular mass in HTX patients (Table 1).

Basic clinical characteristics of HTX patients are presented in Table 2. About $51 \%$ of patients were transplanted due to end-stage heart failure with nonischemic etiology and the operation was performed at a mean age of 51 years. To investigate the potential effects of perioperative circumstances, several hemodynamic and procedural parameters were collected. The median time elapsed after HTX was 226 days, ranging from 8 days to 18 years.

Conventional and 3D echocardiographic parameters of the RV are summarized in Table 3. In terms of conventional linear measurements, RV mid diameter and length were similar, the basal diameter showed enlargement of the RV in HTX patients. Measurements referring to longitudinal shortening showed consequently lower values compared to the control group (TAPSE, s' by tissue Doppler imaging, free wall and septal longitudinal strain). Nevertheless, FAC, which partly incorporates radial function as assessed on a 2D apical four-chamber view, was normal and similar to healthy volunteers in HTX patients $(44 \%$, Table 3). There was no statistically significant difference in terms of end-diastolic and end-systolic RV volumes. RV ejection fraction was
TABLE 1 Baseline characteristics and left ventricular echocardiographic data of heart transplanted patients (HTX) and controls

\begin{tabular}{|c|c|c|c|}
\hline & $\operatorname{HTX}(n=51)$ & Control $(n=30)$ & $P$ value \\
\hline Age, y & $52.3 \pm 10.8$ & $50.1 \pm 13.0$ & .60 \\
\hline Female, n (\%) & $11(22)$ & $11(36)$ & .14 \\
\hline Height, cm & $173.3 \pm 9.4$ & $170.1 \pm 11.7$ & .19 \\
\hline Weight, kg & $74.0 \pm 12.9$ & $70.0 \pm 11.0$ & .16 \\
\hline $\mathrm{BMI}, \mathrm{kg} / \mathrm{m}^{2}$ & $24.6 \pm 4.0$ & $24.1 \pm 2.8$ & .56 \\
\hline $\mathrm{BSA}, \mathrm{m}^{2}$ & $1.9 \pm 0.2$ & $1.8 \pm 0.2$ & .11 \\
\hline SBP, mm Hg & $122.4 \pm 14.0$ & $124.2 \pm 13.1$ & .53 \\
\hline $\mathrm{DBP}, \mathrm{mm} \mathrm{Hg}$ & $79.4 \pm 8.1$ & $74.7 \pm 8.4$ & .41 \\
\hline $\mathrm{HR}, 1 / \min$ & $86.5 \pm 13.1$ & $65.8 \pm 10.4$ & $<.0001$ \\
\hline LV EDV, mL & $100.5 \pm 24.8$ & $95.4 \pm 24.2$ & .46 \\
\hline LV EDVi, $\mathrm{mL} / \mathrm{m}^{2}$ & $53.6 \pm 12.0$ & $52.4 \pm 10.4$ & .66 \\
\hline LV ESV, mL & $38.5 \pm 13.3$ & $35.1 \pm 9.7$ & .31 \\
\hline LV ESVi, mL/m² & $20.5 \pm 6.7$ & $19.2 \pm 4.4$ & .71 \\
\hline LV SV, mL & $62.1 \pm 13.5$ & $58.3 \pm 19.0$ & .53 \\
\hline LV SVi, mL/m² & $33.1 \pm 6.6$ & $33.1 \pm 6.7$ & .99 \\
\hline LV EF, \% & $62.4 \pm 5.8$ & $63.2 \pm 3.4$ & .44 \\
\hline LV GLS, \% & $-19.3 \pm 1.8$ & $-19.1 \pm 2.0$ & .57 \\
\hline LVM, g & $131.6 \pm 22.2$ & $122.5 \pm 20.0$ & .14 \\
\hline LVMi, g/m² & 71.0. \pm 14.0 & $67.8 \pm 9.2$ & .85 \\
\hline
\end{tabular}

BMI, body mass index; BSA, body surface area; DBP, diastolic blood pressure; EDV, end-diastolic volume; EF, ejection fraction; ESV, endsystolic volume; GLS, global longitudinal strain; HR, heart rate; i, indexed to BSA; LV, left ventricle; LVM, left ventricular mass; SBP, systolic blood pressure; SV, stroke volume.

lower in HTX patients; however, it remained within the lower limits of normal range. ${ }^{14}$ Correspondingly, stroke volume and stroke volume index were lower in HTX patients. There were only four patients with moderate tricuspid regurgitation in our HTX group (severe regurgitation was exclusion criterion). Pulmonary arterial systolic pressure was higher in the transplanted cohort than in controls (Table 3).

Figure 1 depicts our results regarding the relative contribution of longitudinal, radial, and anteroposterior wall motions to global RV function. In line with conventional echocardiographic parameters, longitudinal EF and its ratio to TEF was significantly lower in HTX patients compared to healthy controls. However, REF/TEF ratio was significantly higher in HTX patients compared to controls. AEF value alone was lower in HTX patients, and its ratio to TEF was not significantly different from healthy volunteers (Figure 1). In HTX patients, REF/TEF was significantly higher compared to both LEF/TEF and AEF/ TEF (LEF/TEF vs REF/TEF vs AEF/TEF: $0.27 \pm 0.08$ vs $0.58 \pm 0.10$ vs $0.38 \pm 0.07$, ANOVA, $P<.0001$ ), which confirmed the radial wall motion to be dominant determining global RV function after HTX (Figure 2). On the contrary, in healthy volunteers only AEF/TEF ratio was smaller than LEF/TEF, while REF/TEF and LEF/TEF were similar (LEF/TEF vs REF/TEF vs AEF/TEF: $0.47 \pm 0.07$ vs $0.45 \pm 0.07$ vs $0.41 \pm 0.06$, ANOVA, $P=.0034$ ). 
TABLE 2 Indications for heart transplantation (HTX), peri- and postoperative parameters

\begin{tabular}{|lc|}
\hline & HTX $(\mathrm{n}=51)$ \\
\hline Etiology & \\
\hline Nonischemic DCM, $\mathrm{n}(\%)$ & $26(51)$ \\
\hline Ischemic DCM, $\mathrm{n}(\%)$ & $21(41)$ \\
\hline ARVD/C, $\mathrm{n}(\%)$ & $1(2)$ \\
\hline Other, nonspecified, $\mathrm{n}(\%)$ & $3(6)$ \\
\hline Age at HTX, $\mathrm{y}$ & $50.5 \pm 11.1$ \\
\hline Peri- and postoperative parameters & \\
\hline Preoperative PVR, Wood & $2.73 \pm 1.1$ \\
\hline Cold ischemic time, min & $216.3 \pm 44.3$ \\
\hline Aortic cross-clamping time, min & $106.0 \pm 23.1$ \\
\hline Cardiopulmonary bypass time, min & $197.3 \pm 35.5$ \\
\hline Age of donors, $y$ & $41.3 \pm 11.6$ \\
\hline Gender of donors, female, $\mathrm{n}(\%)$ & $8(16)$ \\
\hline Length of ICU stay, $\mathrm{d}$ & $16.7 \pm 17.0$ \\
\hline Postoperative sildenafil use, $\mathrm{n}(\%)$ & $44(86)$ \\
\hline Sildenafil use at time-point of echocardiography, $\mathrm{n}(\%)$ & $5(10)$ \\
\hline Elapsed time after HTX at time-point of & $226(95-827)$ \\
\hline echocardiography, $\mathrm{d}^{\mathrm{a}}$ & \\
\hline
\end{tabular}

ARVD/C, arrhythmogenic right ventricular dysplasia/cardiomyopathy; DCM, dilated cardiomyopathy; ICU, intensive care unit; PVR, pulmonary vascular resistance.

${ }^{\mathrm{a}}$ Median interquartile range.

In HTX patients, RV TEF assessed by 3D echocardiography correlated with FAC $(r=.762, P<.0001)$, free wall LS $(r=.394, P=.018)$ and septal LS $(r=.430, P=.032)$; however, TAPSE did not. LEF correlated moderately ( $r=.421, P=.0023$ ), while REF strongly with TEF $(r=.767, P<.0001)$ in HTX recipients. We found no association between the perioperative hemodynamic or procedural parameters and the RV functional measurements at follow-up. Similarly, no correlation was established between postoperative sildenafil usage and RV morphology and function.

The time elapsed after HTX showed correlation with RV longitudinal function (time vs TAPSE: $r=.577, P<.0001$; vs free wall LS: $r=.483, P=.0003$; vs septal LS: $r=.492, P=.0002$; vs LEF/TEF $r=.289, P=.0039$ ), on the other hand, it had a negative correlation with the dominance of radial contribution (REF/TEF: $r=-.285$, $P=.042$ ). There was no association between anteroposterior shortening of the RV and time after HTX.

We have also compared our HTX patients within 1 year and over 1 year after transplantation (29 vs 22 patients, respectively). There was no difference between the two groups in terms of 3D volumetric RV parameters (HTX within vs over 1 year; RV EDVi: $51.7 \pm 13.5$ vs $49.7 \pm 11.0 \mathrm{~mL} / \mathrm{m}^{2}, P=.57$; RV ESVi: $27.4 \pm 7.8$ vs $26.9 \pm 6.1 \mathrm{~mL} / \mathrm{m}^{2}, P=.80 ; \mathrm{RV}$ SVi: $24.2 \pm 7.2$ vs $22.7 \pm 7.1 \mathrm{~mL} / \mathrm{m}^{2}$, $P=.45$; RV TEF: $47.1 \pm 6.5$ vs $46.3 \pm 8.3 \%, P=.72$ ). While FAC remained unchanged ( $42.3 \pm 7.8$ vs $46.9 \pm 9.7, P=.075)$, parameters referring to longitudinal shortening showed significant increase in time (TAPSE: $9.0 \pm 3.8$ vs $13.3 \pm 6.1 \mathrm{~mm} ; P=.04$, free wall $L S$ :
TABLE 3 Conventional parameters of the right heart in heart transplant recipients (HTX) vs controls

\begin{tabular}{|c|c|c|c|}
\hline & $\operatorname{HTX}(n=51)$ & Control $(n=30)$ & $P$ value \\
\hline $\mathrm{RV}$ basal diameter, $\mathrm{mm}$ & $34.7 \pm 7.6$ & $27.6 \pm 5.1$ & $<.0001$ \\
\hline $\mathrm{RV}$ mid diameter, mm & $32.1 \pm 7.6$ & $29.1 \pm 5.2$ & .07 \\
\hline RV length, mm & $73.4 \pm 8.1$ & $74.2 \pm 6.6$ & .65 \\
\hline TAPSE, mm & $10.8 \pm 5.2$ & $21.1 \pm 3.7$ & $<.0001$ \\
\hline FAC, $\%$ & $44.2 \pm 8.8$ & $44.1 \pm 4.8$ & .99 \\
\hline PW TDI s', cm/s & $10.3 \pm 2.3$ & $13.9 \pm 2.0$ & $<.0001$ \\
\hline RV Tei Index & $0.5 \pm 0.13$ & $0.36 \pm 0.08$ & $<.0001$ \\
\hline RV Free wall LS, \% & $20.1 \pm 5.3$ & $29.5 \pm 3.7$ & $<.0001$ \\
\hline RV Septal LS, \% & $11.9 \pm 4.9$ & $19.5 \pm 4.0$ & $<.0001$ \\
\hline RV EDV, $\mathrm{mL}$ & $96.3 \pm 27.2$ & $97.3 \pm 23.6$ & .87 \\
\hline $\mathrm{RV}$ EDVi, $\mathrm{mL} / \mathrm{m}^{2}$ & $50.8 \pm 12.3$ & $53.9 \pm 11.8$ & .28 \\
\hline RV ESV, mL & $51.2 \pm 15.1$ & $44.9 \pm 12.5$ & .06 \\
\hline $\mathrm{RV} E S V i, \mathrm{~mL} / \mathrm{m}^{2}$ & $27.2 \pm 7.0$ & $24.8 \pm 6.2$ & .13 \\
\hline RV SV, mL & $45.1 \pm 15.3$ & $52.4 \pm 12.5$ & .03 \\
\hline $\mathrm{RV} \mathrm{SVi}, \mathrm{mL} / \mathrm{m}^{2}$ & $23.6 \pm 7.1$ & $29.1 \pm 4.0$ & .0001 \\
\hline RV TEF, \% & $46.7 \pm 7.2$ & $54.1 \pm 4.0$ & $<.0001$ \\
\hline Moderate TR, n (\%) & $4(8)$ & $0(0)$ & $<.0001$ \\
\hline PASP, mm Hg & $34.2 \pm 7.2$ & $16.1 \pm 5.4$ & $<.0001$ \\
\hline IVC at expiration, mm & $16.2 \pm 4.4$ & $14.2 \pm 5.6$ & .16 \\
\hline
\end{tabular}

EDV, end-diastolic volume; ESV, end-systolic volume; FAC, fractional area change; $i$, indexed to body surface area; IVC, inferior vena cava; LS, longitudinal strain; PASP, pulmonary arterial systolic pressure; PW TDI s', pulsed-wave tissue Doppler imaging systolic velocity; RV, right ventricular; $\mathrm{SV}$, stroke volume; TAPSE, tricuspid annular plane systolic excursion; TEF, total ejection fraction; TR, tricuspid regurgitation.

$-18.2 \pm 3.9$ vs $-22.4 \pm 6.2 \%, P=.0047$; septal LS: $-10.6 \pm 3.8$ vs $13.5 \pm 5.9 \%, P=.037)$. The relative contribution of longitudinal and radial wall motions to global RV function was different: The LEF/TEF ratio was significantly higher $(0.23 \pm 0.08$ vs $0.31 \pm 0.06$, $P=.0002)$, the REF/TEF ratio was significantly lower $(0.62 \pm 0.09$ vs $0.54 \pm 0.10, P=.0039$ ) in patients transplanted over 1 year. On the other hand, there was no significant difference in terms of AEF/ TEF between the groups $(0.37 \pm 0.07$ vs $0.40 \pm 0.07, P=.12)$. We found no correlations between perioperative parameters and RV functional measurements in either subgroup.

Intraobserver and interobserver variability for RV volumes are summarized in Table 4. Intraobserver concordance correlation coefficient values ranged from 0.921 to 0.948 , while interobserver values were lower in some degree. These results are comparable to previous publications using the same software for 3D RV model reconstruction. ${ }^{14}$

\section{4 | DISCUSSION}

The main results of our study are that (i) the longitudinal shortening of the RV is significantly decreased in HTX patients without relevant differences in RV geometry and global function; (ii) this phenomenon is attributable to the supernormal radial motion of the RV free wall, 
LEF (\%)

40

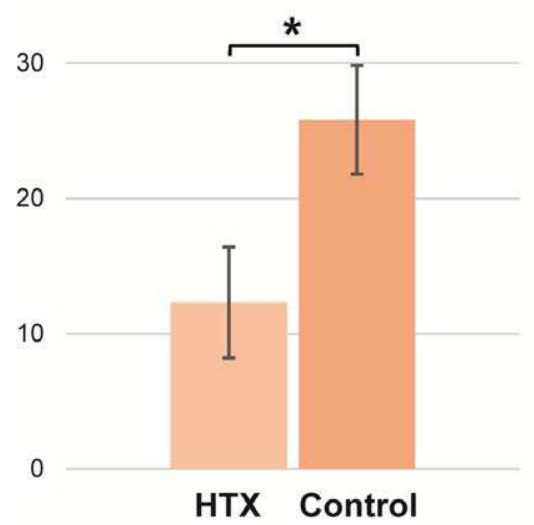

REF (\%)

40

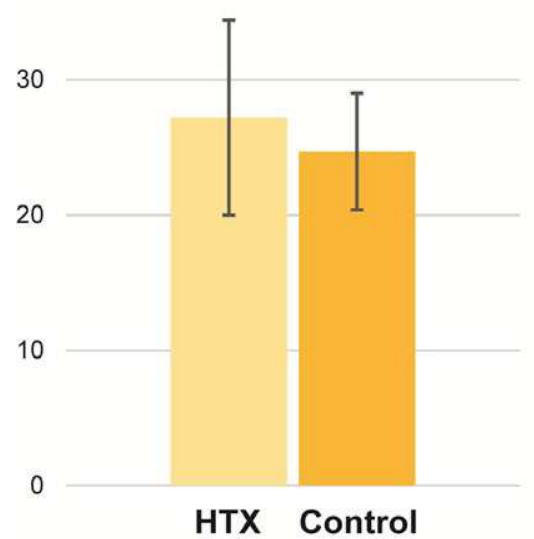

AEF (\%)

40

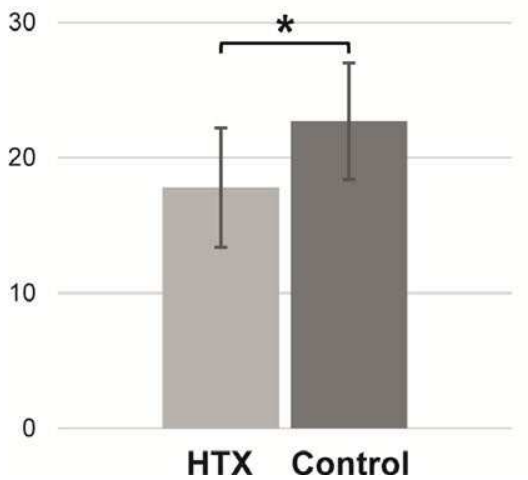

LEF/TEF

0.8

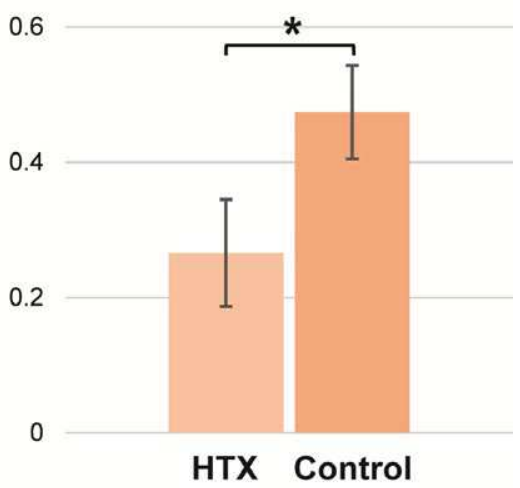

REF/TEF

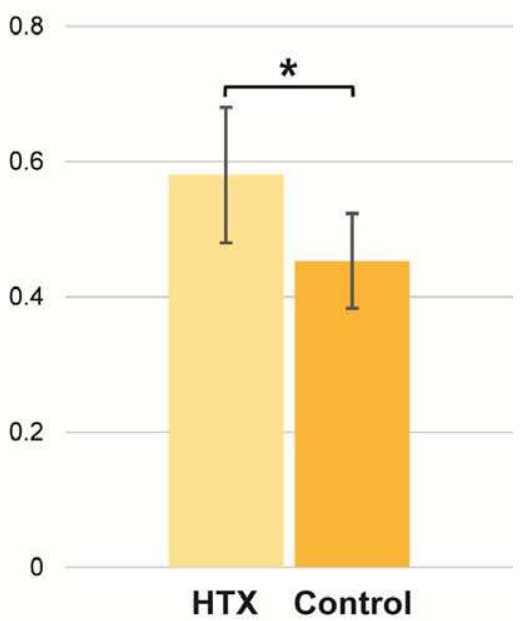

AEF/TEF

0.8

0.6

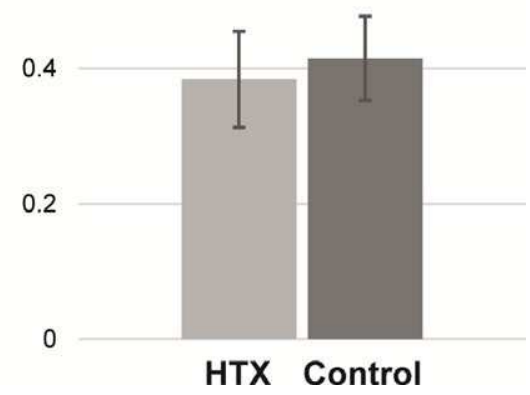

FIGURE 1 Relative contribution of the different wall motion components (longitudinal-LEF, radial-REF, anteroposterior-AEF ejection fraction) to total right ventricular ejection fraction (TEF) in heart transplant recipients (HTX) vs controls. ${ }^{*} P<.05$ 
which maintains RV ejection fraction; (iii) in time, there is a tendency toward the recovery of RV longitudinal shortening in HTX recipients.

About $50 \%$ of cardiac complications and $20 \%$ of mortality are related to RV failure in the early postoperative period after HTX. ${ }^{3,15} \mathrm{RV}$ systolic dysfunction, as assessed by conventional echocardiography, is a common finding in HTX patients. However, the decrease in RV function defined by routine measurements is poorly associated with the clinically manifested right heart failure. The possible cause is that conventional measurements, which refer mainly to the longitudinal shortening of the chamber (TAPSE, $s^{\prime}$ by tissue Doppler imaging), are not accurate to evaluate global RV function in HTX recipients. ${ }^{4,16}$ Echocardiographic data of our cohort of patients showed the same characteristics: Parameters of longitudinal function were decreased not only in the early postoperative period, but also during long-term follow-up. Nevertheless, global function, as assessed by 3D echocardiography, was maintained, which corresponds to previous observations using 3D imaging modalities. ${ }^{6,7}$ TAPSE, the most widely used parameter of RV function, failed to show correlation with 3D derived ejection fraction. There are also several technical issues; however, the altered motion pattern of the structurally complex RV may play a pivotal role in this underestimation. Therefore, to achieve a comprehensive characterization of RV function, other motion directions have to be also taken into consideration.

There are three major mechanisms which contribute to RV pump function: (i) longitudinal shortening by traction of the tricuspid annular plane towards the apex; (ii) the inward (radial) motion of the RV free wall referred as the bellows effect; and (iii) the bulging of the interventricular septum during the left ventricular contraction along with stretching the free wall over the septum. ${ }^{12,17}$ The relative significance of the aforementioned mechanisms may be different in certain cardiovascular conditions. According to our and others' experience by visual estimation, we hypothesized that the radial motion is dominant determining global RV function in HTX patients.

Several previous works suggested the importance of the radial free wall motion; however, 2D imaging modalities failed to robustly delineate this motion direction due to the complex $3 D$ structure of the RV. ${ }^{4,6,18,19}$ Fractional area change represents an important 2D parameter, because it partly incorporates the radial direction by being calculated from the end-diastolic and end-systolic areas of the RV on an apical four-chamber view or even during transesophageal echocardiography. In our study, FAC was similar in HTX patients and healthy subjects, while correlating well with ejection fraction. However, FAC suffers from the inherent $2 \mathrm{D}$ nature of its calculation, referring to a single plane of the large RV free wall surface. Three-dimensional echocardiography provides precise geometric information on the RV and also permits the measurement of global function (ie, ejection fraction), which was only achievable by cardiac MRI. ${ }^{20}$ It has been shown that 3D echocardiography-derived ejection fraction is a useful marker of functional status after HTX. ${ }^{21}$ Moreover, further analysis of the 3D models enables to separately quantify the different wall motion directions and evaluate the relative contribution of each component. ${ }^{12}$ Using this approach, our results confirmed the dominance of radial wall motion in HTX patients. REF/TEF ratio was supernormal, while
LEF/TEF was decreased similarly to other parameters of longitudinal function (Figure 2). In the control group, the relative contribution of longitudinal and radial wall motions was nearly the same.

Furthermore, the results of our cohort correspond to previous literature that RV longitudinal shortening remains decreased throughout long-term follow-up. ${ }^{6,21}$ Patients transplanted over 1 year still presented with decreased longitudinal function. However, a slow recovery is suggested to be present, while global function and geometry remain unchanged. We found correlations between TAPSE, longitudinal strain, LEF/TEF, and the time elapsed after HTX. Therefore, the instantaneous dominance of the radial wall motion after transplantation may be shifted back toward longitudinal shortening in time. The anteroposterior motion of the RV may mainly reflect the ventricular interdependence: Left ventricular contraction stretches the RV free wall insertion points resulting in a considerable contribution to RV function. The relative contribution of anteroposterior shortening to global function was unaffected by time, which may be related to the maintained left ventricular function.

There are several underlying mechanisms which may be the causes of this functional change. First and foremost, the elevated pulmonary pressure of the recipient may be a factor. Beyond the preexistent pulmonary hypertension, cardiopulmonary bypass also results in a transient pulmonary vasoconstriction. ${ }^{22}$ It is also known that pulmonary hypertension is a significant risk factor of acute right-sided failure and also a predictor of long-term survival. ${ }^{15,23}$ In the early postoperative period, increased afterload results in dilation of the RV. ${ }^{24}$ However, pulmonary vascular resistance decreases after the procedure and only slightly elevated pressures are expected later, even in cases of severe preoperative pulmonary hypertension. ${ }^{24-26}$ Therefore, we should be cautious highlighting only pulmonary pressures regarding RV functional shift on a long-term follow-up. In primary pulmonary hypertension patients, a close relationship can be observed between pulmonary vascular resistance and various RV functional parameters. ${ }^{27}$ Moreover, these patients show maintained longitudinal RV shortening for a long time and the loss of radial function is suggested to be an early marker of the disease. ${ }^{28,29}$ These findings are not concordant with what is seen in HTX patients.

Beside hemodynamics, (patho)physiological changes associated with brain death of the donor and also organ preservation may contribute to the functional shift. ${ }^{30,31}$ It has been shown that the loss of pericardial constraint due to the incomplete closure of the pericardial sac and mismatch in the donor-recipient heart size has significant impact on cardiac mechanics. ${ }^{32}$ According to experimental simulations, the intact pericardial sac may reduce radial contraction of the ventricles with a concomitant increase in the longitudinal function. ${ }^{33}$ Clinical studies also support that the deterioration of $\mathrm{RV}$ longitudinal function is concurrent with the pericardial incision. ${ }^{34} \mathrm{RV}$ longitudinal shortening measured by TAPSE is reported to be reduced in cardiac surgery patients as well, despite normal ejection fraction, therefore, the same phenomenon partly coexists after every cardiac surgery. ${ }^{18,35}$ However, the magnitude of longitudinal functional loss seems to be smaller and the recovery appears much faster in these patient populations. ${ }^{36-38}$ Previous studies reported the similar nature and extent of RV functional shift in cardiac 


\section{Right ventricle of a heart transplant recipient}
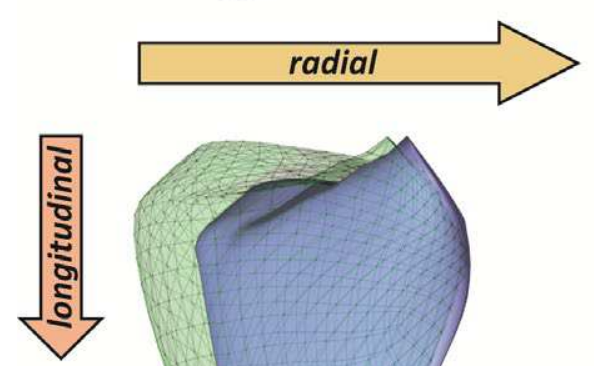

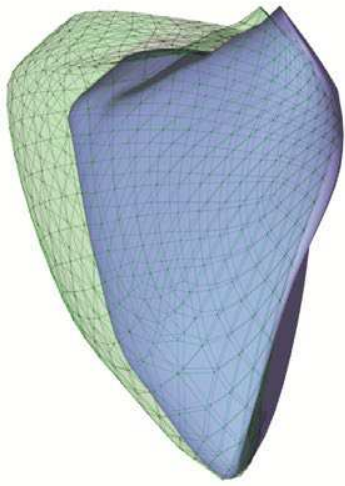

global function

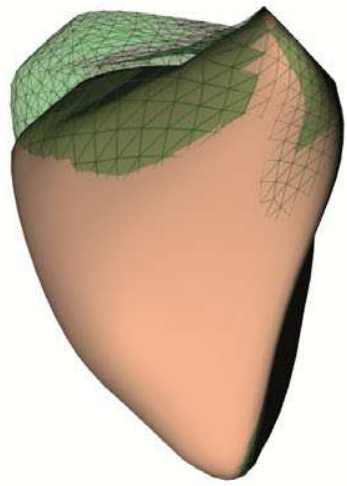

longitudinal motion only

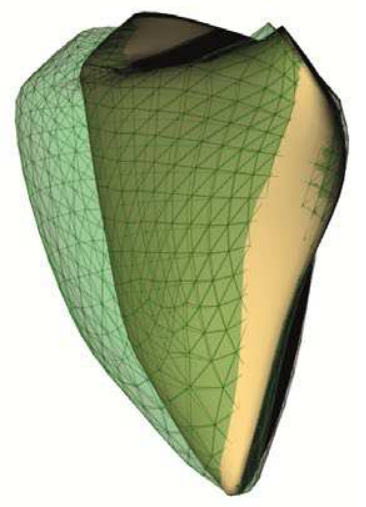

radial motion only

\section{Right ventricle of a healthy volunteer}

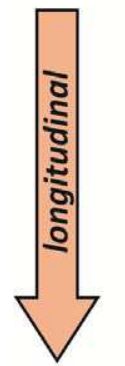

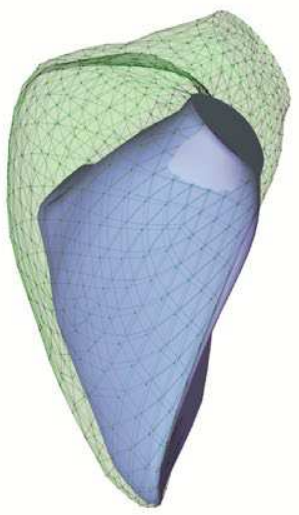

global function

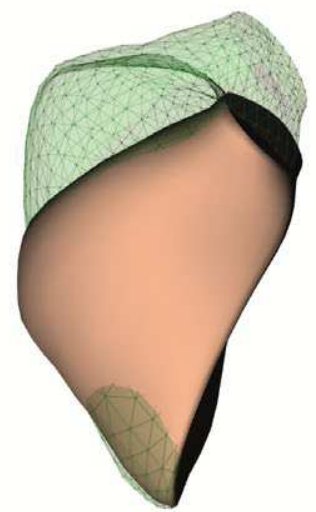

longitudinal motion only

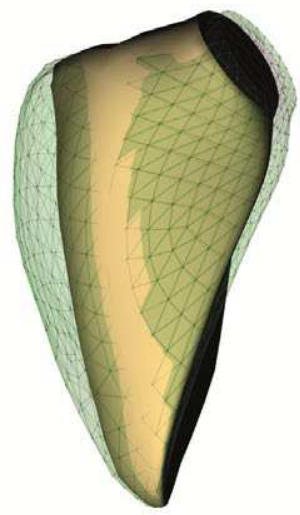

radial motion only

FIGURE 2 Representative examples of right ventricular motion pattern in a heart transplant recipient vs a healthy volunteer. Green mesh represents end-diastolic volume, and the blue surface is the end-systolic volume with all motion directions enabled. By decomposing the motion of the three-dimensional right ventricular model, the different anatomically relevant wall motion directions can be separately quantified. The radial motion is supernormal, and the longitudinal is decreased in the heart transplanted patient compared to the healthy volunteer. Orange surface represents the volume loss at end-systole generated by only the longitudinal motion. Yellow surface represents the volume loss at endsystole generated by only the radial motion

surgery patients with or without pericardial repair, suggesting that the loss of pericardial constraint may not be solely responsible for the altered RV mechanics. ${ }^{39}$ We should also mention the potential detrimental effects of immunosuppressive regimens in this regard. ${ }^{40}$ Beyond the aforementioned potential causes, loss of the substantial innervation of the RV myocardium may be an other important factor resulting in complex functional changes of the RV. ${ }^{41}$ However, it has been suggested that the transplanted heart can be reinnervated by both sympathetic and parasympathetic fibers. ${ }^{42,43}$ Hypothetically, the slow recovery of longitudinal function can be a result of reinnervation as well. Of note, we may expect differences between the surgical techniques applied during HTX, which can result in different geometry and function of the right heart. ${ }^{44}$

\section{1 | Limitations}

Despite the fair case number, an evident limitation of our study is its retrospective, cross-sectional approach. The patient population is 
TABLE 4 Intra- and interobserver variability of RV threedimensional echocardiography derived volumes. Lin's concordance correlation coefficient values

\begin{tabular}{lll} 
& $\begin{array}{l}\text { Intraobserver } \\
\text { variability }(95 \% \mathrm{Cl})\end{array}$ & $\begin{array}{l}\text { Interobserver } \\
\text { variability }(95 \% \mathrm{Cl})\end{array}$ \\
\hline RV EDV & $0.921(0.821-0.966)$ & $0.901(0.792-0.954)$ \\
\hline RV ESV & $0.948(0.876-0.979)$ & $0.925(0.831-0.967)$ \\
\hline $\begin{array}{c}\text { ESV (longitudinal } \\
\text { only) }\end{array}$ & $0.923(0.827-0.967)$ & $0.887(0.747-0.952)$ \\
\hline ESV (radial only) & $0.934(0.845-0.973)$ & $0.913(0.838-0.954)$ \\
\hline
\end{tabular}

$\mathrm{RV}$, right ventricle; EDV, end-diastolic volume; ESV, end-systolic volume.

broad in terms of time elapsed after transplantation, and therefore, the correlations of functional parameters vs time could be demonstrated. On the other hand, we were unable to show the potential significance of the perioperative hemodynamic and procedural parameters. We have initialized our prospective study to better characterize the timing and causes of this functional shift mechanism. Complete exclusion of higher grade rejection episodes and chronic allograft vasculopathy (which can deteriorate biventricular myocardial mechanics) is difficult, especially in patients transplanted for a longer time. ${ }^{45-47}$ Regular biopsies are discontinued after 1 year according to our institutional protocol, while chronic allograft vasculopathy is assessed on a yearly basis using invasive or CT coronary angiography. Of note, the reported TAPSE values are calculated by the dedicated 3D software, which results in lower absolute values compared to the M-mode method. This may limit comparability to previous publications, but fits better to our retrospective study design gaining better generalizability.

\section{2 | Conclusions}

After HTX, the radial motion of RV free wall compensates the decreased longitudinal shortening to maintain RV ejection fraction. In time, longitudinal function may recover. Three-dimensional echocardiography may be a useful method in everyday clinical practice to accurately measure global RV function in HTX patients. If 3D analysis is not available, a detailed 2D echocardiographic assessment is necessary involving such measurements, which refer to the radial motion of the RV. Prospective studies are needed to better characterize the timing and causes of RV functional shift and to determine the potential predictive value of the novel RV parameters on the outcome of heart transplant recipients.

\section{CONFLICT OF INTEREST}

All authors have nothing to disclose regarding this work.

\section{AUTHORS' CONTRIBUTIONS}

Bálint Lakatos contributed to literature search, data collection, data analysis, data interpretation, writing. Márton Tokodi performed literature search, data analysis, data interpretation, figure preparation, writing. Alexandra Assabiny performed data analysis, data interpretation, writing. Zoltán Tősér performed software development, data analysis, data interpretation, reviewing. Annamária Kosztin, Alexandra Doronina, Kinga Koritsánszky, and Viktor Berzsenyi performed data collection, data analysis, data interpretation, reviewing. Kristóf Rácz performed study design, data collection, data analysis, data interpretation, reviewing. Endre Németh and Balázs Sax performed study design, data interpretation, reviewing. Attila Kovács contributed to study design, literature search, data collection, data interpretation, writing. Béla Merkely performed study design, funding, data interpretation, reviewing.

\section{ORCID}

Bálint Károly Lakatos (iD http://orcid.org/0000-0002-7627-5620

Endre Németh iD http://orcid.org/0000-0001-6033-4297

Attila Kovács (iD http://orcid.org/0000-0003-2320-6434

Béla Merkely (iD http://orcid.org/0000-0001-6514-0723

\section{REFERENCES}

1. Yancy CW, Jessup M, Bozkurt B, et al. 2013 ACCF/AHA guideline for the management of heart failure: a report of the American College of Cardiology Foundation/American Heart Association Task Force on Practice Guidelines. J Am Coll Cardiol. 2013;62:e147-e239.

2. Ponikowski P, Voors AA, Anker SD, et al. 2016 ESC Guidelines for the diagnosis and treatment of acute and chronic heart failure: The Task Force for the diagnosis and treatment of acute and chronic heart failure of the European Society of Cardiology (ESC). Developed with the special contribution of the Heart Failure Association (HFA) of the ESC. Eur J Heart Fail. 2016;18:891-975.

3. Yusen RD, Edwards LB, Dipchand Al, et al. The Registry of the International Society for Heart and Lung Transplantation: thirty-third adult lung and heart-lung transplant report - 2016; focus theme: primary diagnostic indications for transplant. J Heart Lung Transplant. 2016;35:1170-1184.

4. Badano LP, Miglioranza MH, Edvardsen T, et al. European Association of Cardiovascular Imaging/Cardiovascular Imaging Department of the Brazilian Society of Cardiology recommendations for the use of cardiac imaging to assess and follow patients after heart transplantation. Eur Heart J Cardiovasc Imaging. 2015;16:919-948.

5. Kobashigawa J, Zuckermann A, Macdonald P, et al. Report from a consensus conference on primary graft dysfunction after cardiac transplantation. J Heart Lung Transplant. 2014;33:327-340.

6. D'Andrea A, Riegler L, Nunziata L, et al. Right heart morphology and function in heart transplantation recipients. J Cardiovasc Med (Hagerstown). 2013;14:648-658.

7. Bacal F, Pires PV, Moreira LF, et al. Normalization of right ventricular performance and remodeling evaluated by magnetic resonance imaging at late follow-up of heart transplantation: relationship between function, exercise capacity and pulmonary vascular resistance. J Heart Lung Transplant. 2005;24:2031-2036.

8. Lang RM, Badano LP, Mor-Avi V, et al. Recommendations for cardiac chamber quantification by echocardiography in adults: an update from the American Society of Echocardiography and the European Association of Cardiovascular Imaging. J Am Soc Echocardiogr. 2015;28:1-39.e14.

9. Rudski LG, Lai WW, Afilalo J, et al. Guidelines for the echocardiographic assessment of the right heart in adults: a report from the 
American Society of Echocardiography endorsed by the European Association of Echocardiography, a registered branch of the European Society of Cardiology, and the Canadian Society of Echocardiography. J Am Soc Echocardiogr. 2010;23:685-713; quiz 786-788.

10. Lancellotti P, Tribouilloy C, Hagendorff A, et al. Recommendations for the echocardiographic assessment of native valvular regurgitation: an executive summary from the European Association of Cardiovascular Imaging. Eur Heart J Cardiovasc Imaging. 2013;14:611-644.

11. Mosteller RD. Simplified calculation of body-surface area. N Engl J Med. 1987;317:1098.

12. Lakatos B, Tösér Z, Tokodi M, et al. Quantification of the relative contribution of the different right ventricular wall motion components to right ventricular ejection fraction: the ReVISION method. Cardiovasc Ultrasound. 2017;15:8.

13. Ho SY, Nihoyannopoulos P. Anatomy, echocardiography, and normal right ventricular dimensions. Heart. 2006;92(suppl 1):i2-i13.

14. Maffessanti F, Muraru D, Esposito R, et al. Age-, body size-, and sex-specific reference values for right ventricular volumes and ejection fraction by three-dimensional echocardiography: a multicenter echocardiographic study in 507 healthy volunteers. Circ Cardiovasc Imaging. 2013;6:700-710.

15. Stobierska-Dzierzek B, Awad H, Michler RE. The evolving management of acute right-sided heart failure in cardiac transplant recipients. J Am Coll Cardiol. 2001;38:923-931.

16. Hyden M, Anchan RK, High R, et al. A comparison of two-dimensional echocardiography parameters with cardiac magnetic resonance imaging in the assessment of right ventricular function post orthotopic heart transplantation. J Heart Lung Transplant. 2017;36:S156-S157.

17. Badano LP, Ginghina C, Easaw J, et al. Right ventricle in pulmonary arterial hypertension: haemodynamics, structural changes, imaging, and proposal of a study protocol aimed to assess remodelling and treatment effects. Eur J Echocardiogr. 2010;11:27-37.

18. Raina A, Vaidya A, Gertz ZM, Chambers S, Forfia PR. Marked changes in right ventricular contractile pattern after cardiothoracic surgery: implications for post-surgical assessment of right ventricular function. J Heart Lung Transplant. 2013;32:777-783.

19. Sakuma M, Ishigaki $H$, Komaki $K$, et al. Right ventricular ejection function assessed by cineangiography - importance of bellows action. Circ J. 2002;66:605-609.

20. Muraru D, Spadotto V, Cecchetto A, et al. New speckle-tracking algorithm for right ventricular volume analysis from three-dimensional echocardiographic data sets: validation with cardiac magnetic resonance and comparison with the previous analysis tool. Eur Heart J Cardiovasc Imaging. 2016;17:1279-1289.

21. Clemmensen TS, Eiskjær H, Løgstrup BB, et al. Echocardiographic assessment of right heart function in heart transplant recipients and the relation to exercise hemodynamics. Transpl Int. 2016;29:909-920.

22. Nyhan DP, Redmond JM, Gillinov AM, Nishiwaki KIMITOSHI, Murray PA. Prolonged pulmonary vascular hyperreactivity in conscious dogs after cardiopulmonary bypass. J Appl Physiol (1985). 1994;77:1584-1590.

23. Erickson KW, Costanzo-Nordin MR, O'Sullivan EJ, et al. Influence of preoperative transpulmonary gradient on late mortality after orthotopic heart transplantation. J Heart Transplant. 1990;9:526-537.

24. Bhatia SJ, Kirshenbaum JM, Shemin RJ, et al. Time course of resolution of pulmonary hypertension and right ventricular remodeling after orthotopic cardiac transplantation. Circulation. 1987;76:819-826.

25. Bourge RC, Kirklin JK, Naftel DC, White C, Mason DA, Epstein AE. Analysis and predictors of pulmonary vascular resistance after cardiac transplantation. J Thorac Cardiovasc Surg. 1991;101:432-444; discussion 444-445.

26. Tedford RJ, Beaty CA, Mathai SC, et al. Prognostic value of the pre-transplant diastolic pulmonary artery pressure-to-pulmonary capillary wedge pressure gradient in cardiac transplant recipients with pulmonary hypertension. J Heart Lung Transplant. 2014;33:289-297.

27. Murata M, Tsugu T, Kawakami T, et al. Prognostic value of threedimensional echocardiographic right ventricular ejection fraction in patients with pulmonary arterial hypertension. Oncotarget. 2016;7:86781-86790.

28. Kind T, Mauritz GJ, Marcus JT, van de Veerdonk M, Westerhof N, Vonk-Noordegraaf A. Right ventricular ejection fraction is better reflected by transverse rather than longitudinal wall motion in pulmonary hypertension. J Cardiovasc Magn Reson. 2010;12:35.

29. Brown SB, Raina A, Katz D, Szerlip M, Wiegers SE, Forfia PR. Longitudinal shortening accounts for the majority of right ventricular contraction and improves after pulmonary vasodilator therapy in normal subjects and patients with pulmonary arterial hypertension. Chest. 2011;140:27-33.

30. Bittner HB, Chen EP, Milano CA, et al. Myocardial beta-adrenergic receptor function and high-energy phosphates in brain death-related cardiac dysfunction. Circulation. 1995;92(9 suppl):I1472-II478.

31. Ferrera R, Hadour G, Tamion F, et al. Brain death provokes very acute alteration in myocardial morphology detected by echocardiography: preventive effect of beta-blockers. Transpl Int. 2011;24:300-306.

32. Schwarz K, Singh S, Dawson D, Frenneaux MP. Right ventricular function in left ventricular disease: pathophysiology and implications. Heart Lung Circ. 2013;22:507-511.

33. Fritz T, Wieners C, Seemann G, Steen H, Dössel O. Simulation of the contraction of the ventricles in a human heart model including atria and pericardium. Biomech Model Mechanobiol. 2014;13: 627-641.

34. Unsworth B, Casula RP, Kyriacou AA, et al. The right ventricular annular velocity reduction caused by coronary artery bypass graft surgery occurs at the moment of pericardial incision. Am Heart J. 2010;159:314-322.

35. Tamborini G, Muratori M, Brusoni $D$, et al. Is right ventricular systolic function reduced after cardiac surgery? A two- and three-dimensional echocardiographic study. Eur J Echocardiogr. 2009;10:630-634.

36. Maffessanti F, Gripari P, Tamborini G, et al. Evaluation of right ventricular systolic function after mitral valve repair: a two-dimensional Doppler, speckle-tracking, and three-dimensional echocardiographic study. J Am Soc Echocardiogr. 2012;25:701-708.

37. Schuuring MJ, Bolmers PP, Mulder BJ, et al. Right ventricular function declines after cardiac surgery in adult patients with congenital heart disease. Int J Cardiovasc Imaging. 2012;28:755-762.

38. Hedman A, Alam M, Zuber E, Nordlander R, Samad BA. Decreased right ventricular function after coronary artery bypass grafting and its relation to exercise capacity: a tricuspid annular motion-based study. J Am Soc Echocardiogr. 2004;17:126-131.

39. Lindqvist P, Holmgren A, Zhao Y, Henein MY. Effect of pericardial repair after aortic valve replacement on septal and right ventricular function. Int J Cardiol. 2012;155:388-393.

40. Peura JL, Zile MR, Feldman DS, et al. Effects of conversion from cyclosporine to tacrolimus on left ventricular structure in cardiac allograft recipients. J Heart Lung Transplant. 2005;24:1969-1972.

41. Wink J, de Wilde RB, Wouters PF, et al. Thoracic epidural anesthesia reduces right ventricular systolic function with maintained ventricular-pulmonary coupling. Circulation. 2016;134:1163-1175.

42. Buendia-Fuentes F, Almenar L, Ruiz C, et al. Sympathetic reinnervation 1 year after heart transplantation, assessed using iodine-123 metaiodobenzylguanidine imaging. Transplant Proc. 2011;43:2247-2248.

43. Bernardi L, Bianchini B, Spadacini G, et al. Demonstrable cardiac reinnervation after human heart transplantation by carotid baroreflex modulation of RR interval. Circulation. 1995;92:2895-2903.

44. Beniaminovitz A, Savoia MT, Oz M, et al. Improved atrial function in bicaval versus standard orthotopic techniques in cardiac transplantation. Am J Cardiol. 1997;80:1631-1635. 
45. Clemmensen TS, Løgstrup BB, Eiskjær H, Poulsen SH. Serial changes in longitudinal graft function and implications of acute cellular graft rejections during the first year after heart transplantation. Eur Heart J Cardiovasc Imaging. 2016;17:184-193.

46. Sera F, Kato TS, Farr M, et al. Left ventricular longitudinal strain by speckle-tracking echocardiography is associated with treatmentrequiring cardiac allograft rejection. J Card Fail. 2014;20:359-364.

47. Pichler P, Binder T, Höfer P, et al. Two-dimensional speckle tracking echocardiography in heart transplant patients: three-year follow-up of deformation parameters and ejection fraction derived from transthoracic echocardiography. Eur Heart J Cardiovasc Imaging. 2012;13:181-186.

How to cite this article: Lakatos BK, Tokodi M, Assabiny A, et al. Dominance of free wall radial motion in global right ventricular function of heart transplant recipients. Clin Transplant. 2018;32:e13192. https://doi.org/10.1111/ctr.13192 